\title{
Dose and Schedule Selection of the Oral Proteasome Inhibitor Ixazomib in Relapsed/Refractory Multiple Myeloma: Clinical and Model-Based Analyses
}

\author{
Neeraj Gupta ${ }^{1}$ (D) Huyuan Yang ${ }^{1} \cdot$ Michael J. Hanley ${ }^{1} \cdot$ Steven Zhang ${ }^{1} \cdot$ Rachael Liu $^{1}$ • \\ Shaji Kumar $^{2}$ - Paul G. Richardson ${ }^{3}$. Tomas Skacel ${ }^{1,4} \cdot$ Karthik Venkatakrishnan $^{1}$
}

Published online: 12 August 2017

(C) The Author(s) 2017. This article is an open access publication

\begin{abstract}
Background The oral proteasome inhibitor ixazomib has been approved by regulatory authorities around the world, including in the United States and the European Union, for the treatment of patients with multiple myeloma (MM) who have received at least one prior therapy, based on the pivotal phase III TOURMALINE-MM1 study.

Objective The objective of this study was to quantitatively characterize the benefit-risk profile of ixazomib in relapsed/ refractory MM in support of the approved dose and schedule. Methods We report early-phase study data and exposure-response analyses of TOURMALINE-MM1 data that support the selection of the recommended ixazomib dose and schedule.

Results Single-agent ixazomib studies showed a favorable efficacy/safety profile with weekly versus twice-weekly dosing; a phase I/II study of ixazomib in combination with lenalidomide and dexamethasone (IRd) identified a
\end{abstract}

Electronic supplementary material The online version of this article (doi:10.1007/s11523-017-0524-3) contains supplementary material, which is available to authorized users.

Neeraj Gupta

Neeraj.Gupta@takeda.com

1 Millennium Pharmaceuticals, Inc., a wholly owned subsidiary of Takeda Pharmaceutical Company Limited, Cambridge, MA, USA

2 Division of Hematology, Mayo Clinic, Rochester, MN, USA

3 Dana-Farber Cancer Institute, Boston, MA, USA

4 Department of Hematology, Charles University General Hospital, Prague, Czech Republic weekly ixazomib dose that offered an acceptable efficacy/ safety profile. In IRd exposure-response analyses from TOURMALINE-MM1, ixazomib systemic exposure was not a significant predictor of progression-free survival or probability of response. Significant associations were observed between ixazomib exposure and the probability of grade $\geq 3$ anemia and thrombocytopenia, and grade $\geq 2$ diarrhea, fatigue, nausea, peripheral neuropathy, and rash. Additionally, higher ixazomib exposure was associated with lower lenalidomide relative dose intensity.

Conclusions These analyses support a favorable benefit-risk profile for weekly ixazomib $4.0 \mathrm{mg}$ on days 1,8 , and 15 of 28-day cycles, which was selected for the phase III TOURMALINE registration program.

Trial Registration Numbers ClinicalTrials.gov NCT00932698, NCT00963820, NCT01217957, NCT01564537

\section{Key Points}

Weekly ixazomib $4.0 \mathrm{mg}$ in combination with lenalidomide and dexamethasone (IRd) provides an acceptable efficacy/safety profile without negatively impacting lenalidomide relative dose intensity

Exposure-PFS/response analyses show that the efficacy benefit of IRd was consistent across the range of exposures achieved using the weekly $4.0 \mathrm{mg}$ dose of ixazomib

Exposure-safety analyses demonstrated a relationship between ixazomib exposure and several adverse events, but these were readily manageable and not associated with major clinical complications or substantial rates of discontinuations 


\section{Introduction}

Ixazomib, the first oral proteasome inhibitor [1], has been approved by multiple regulatory authorities, including in the United States and the European Union, in combination with lenalidomide and dexamethasone (Rd), for the treatment of patients with multiple myeloma (MM) who have received at least one prior therapy $[2,3]$. Approval was based on the global, randomized, double-blind, placebo-controlled, phase III TOURMALINE-MM1 study in relapsed/refractory MM [4], in which an all-oral triplet regimen of ixazomib plus $\mathrm{Rd}$ (IRd) demonstrated a significant and clinically meaningful progression-free survival (PFS) benefit with limited additional toxicity compared with placebo plus Rd (placebo-Rd).

The evaluation and optimization of dose is critical for a successful oncology drug development program. Dose selection for oncology drugs has traditionally been based on determination of the maximum tolerated dose (MTD), often using a $3+3$ dose-escalation strategy [5-8]. However, there are limitations to this approach, and the importance of fully understanding the relationship between pharmacokinetics, pharmacodynamics, safety, and efficacy, both during and after early-phase studies, is now recognized $[6,7,9]$. Several dose-selection approaches have been proposed [5-8], with exposure-response analyses (exposure-efficacy analyses, exposure-safety analyses, or both) increasingly commonly used for oncology drugs [5-8].

The TOURMALINE-MM1 IRd regimen utilized weekly oral dosing of ixazomib $4.0 \mathrm{mg}$ on days 1,8 , and 15 of 28 day cycles. Pharmacokinetic analyses have demonstrated that this dose and schedule is appropriate for a broad patient population, including patients with mild-to-moderate renal impairment, patients with mild hepatic impairment [10, 11], and East Asian patients with relapsed/refractory MM [12]. Moreover, a population pharmacokinetic analysis of earlyphase data demonstrated the feasibility of using fixed ixazomib dosing instead of body surface area (BSA)-based dosing [11]. Other clinical studies of ixazomib, alone and in combination, have evaluated twice-weekly dosing and higher doses in different MM patient populations [13-18].

In the context of the tolerability of the IRd regimen in the TOURMALINE-MM1 study, and the limited additional toxicity compared to the placebo regimen, we report the evaluations of early-phase studies and an exposure-response analysis of data from the TOURMALINE-MM1 study that provide the rationale for the selection of the ixazomib dose and schedule for patients with relapsed/refractory MM.

\section{Methods}

\subsection{Analysis of Early-Phase Studies of Ixazomib}

Key efficacy and safety data were compared from two phase I studies of single-agent ixazomib (NCT00932698,
NCT00963820), employing twice-weekly [18] and weekly administration [13], in patients with relapsed or relapsed and refractory MM. Data were compared between patients in the MTD-expansion cohort for each study; in the twice-weekly study, the MTD was $2.0 \mathrm{mg} / \mathrm{m}^{2}$ (equivalent on average to a fixed dose of $3.7 \mathrm{mg}$ ) on days 1,4 , 8 , and 11 of 21-day cycles, and in the weekly study, the MTD was $2.97 \mathrm{mg} / \mathrm{m}^{2}$ (equivalent on average to a fixed dose of $5.5 \mathrm{mg}$ ) on days 1,8 , and 15 of 28-day cycles. Efficacy, safety, and dose intensity data were also evaluated from a phase I/II study of IRd in patients with newly diagnosed MM (NCT01217957) [14], in which ixazomib doses of up to $3.95 \mathrm{mg} / \mathrm{m}^{2}$ were investigated; the MTD was $2.97 \mathrm{mg} / \mathrm{m}^{2}$ on days 1,8 , and 15 of 28 -day cycles.

\subsection{Exposure-Response Analysis of Data from Phase III Study}

The pivotal phase III TOURMALINE-MM1 study (NCT01564537) compared the efficacy and safety of IRd versus placebo-Rd in 722 patients with relapsed/refractory MM who had received one to three prior therapies [4]. The primary endpoint of the study was PFS. An exposure-response analysis was conducted to explore the relationship between ixazomib systemic exposure and PFS, clinical response rates, selected common/overlapping adverse events (AEs) ascribed to ixazomib, and time to first dose reduction of ixazomib in patients treated with IRd. Additionally, the relationship between ixazomib exposure and lenalidomide relative dose intensity (RDI) was evaluated. All PFS, clinical response, safety, and RDI data were derived from the first interim analysis of TOURMALINE-MM1, which was the final statistical analysis for PFS, after a median follow-up of $\sim 15$ months. Assessments of response and disease progression in TOURMALINE-MM1 were conducted by an independent review committee, using central laboratory results and the International Myeloma Working Group 2011 criteria [19]. AEs were graded according to National Cancer Institute Common Terminology Criteria for Adverse Events version 4.03.

For the exposure-response analyses, data from a population pharmacokinetic model for ixazomib [10] were used to determine the time-averaged systemic exposure metric for ixazomib. The area under the plasma ixazomib concentration-time curve (AUC) per day (AUC/day) was derived for each patient using the available ixazomib dosing information, individual patient oral clearance $(\mathrm{CL} / \mathrm{F})$ values from the population pharmacokinetic model, and the time to the occurrence of the first event under evaluation, i.e., progression/death in the analysis of the relationship for PFS, confirmed best clinical response, maximum grade of $\mathrm{AE}$ (grade $\geq 3$ for hematologic AEs, grade $\geq 2$ for non-hematologic AEs), or first ixazomib dose reduction. For calculation of total ixazomib dose and total time to event, total time included the number of days from day 
1 , cycle 1 , until the event, including the day of the event, and total dose included all ixazomib doses received before the event. For events that occurred on ixazomib dosing days, the dose on the event day was not included in the total dose calculation. In the exposure-efficacy analyses, for events that occurred after a patient's last dosing cycle, the time to event was set as the time to the end of the last dosing cycle. In the exposure-safety analyses, for events that happened on day 1 , cycle 1 , the time to event was set as the full cycle length of 28 days. For each analysis, only patients with both pharmacokinetic and response (efficacy or safety) information were included; 347 of the 360 patients (96\%) in the IRd intent-to-treat (ITT) population had sufficient pharmacokinetic data to estimate $\mathrm{CL} / \mathrm{F}$ and all 347 patients were included in the exposure-safety and exposure-time to first dose reduction analyses. Five patients were excluded from the exposure-PFS analysis $(n=342)$ due to being censored at randomization, and nine patients were excluded from the exposure-clinical response analysis $(n=338)$ as they were not response-evaluable. Patients who received at least one dose of study drug, had measurable disease at baseline, and had at least one post-baseline response assessment were considered response-evaluable.

\subsection{Statistical Methods for Exposure-Response Analyses}

For the exposure-response analyses of TOURMALINEMM1 data, exposure-PFS and exposure-dose reduction relationships were characterized by Cox proportional hazards models relating exposure to the hazard of PFS or first ixazomib dose reduction, respectively. Kaplan-Meier plots of PFS and time to first ixazomib dose reduction were generated and stratified by ixazomib exposure quartiles. The exposure-clinical response and exposure-safety analyses used logistic regression models to determine the relationship between time-averaged ixazomib exposure and probability of achieving best response or occurrence of an AE. For the exposure-lenalidomide RDI analysis, the proportion of patients with a lenalidomide RDI of $\geq 60 \%$ was determined for each ixazomib exposure quartile and the relationship was analyzed using logistic regression. Full details of the statistical methods employed are provided in the Supplementary Methods (Electronic Supplementary Material online resource).

\section{Results}

\subsection{Early-Phase Studies}

In the two phase I studies of single-agent ixazomib [13, 18], 60 patients were enrolled in each study, with 40 treated in the MTD-expansion cohort (ixazomib dose: $2.0 \mathrm{mg} / \mathrm{m}^{2}$ ) in the study of twice-weekly ixazomib, and 31 in the MTD-
Table 1 Summary of key efficacy and safety parameters in the maximum tolerated dose-expansion cohorts of the two phase I studies of twice-weekly and weekly single-agent ixazomib in patients with relapsed/refractory multiple myeloma

\begin{tabular}{lll}
\hline Parameter & $\begin{array}{l}\text { Twice-weekly study, } \\
\text { ixazomib dose } \\
2.0 \mathrm{mg} / \mathrm{m}^{2}[\mathrm{n}(\%)]\end{array}$ & $\begin{array}{l}\text { Weekly study, } \\
\text { ixazomib dose } \\
2.97 \mathrm{mg} / \mathrm{m}^{2}[\mathrm{n}(\%)]\end{array}$ \\
\hline $\begin{array}{l}\text { Response-evaluable } \\
\text { patients (n) }\end{array}$ & 39 & 30 \\
$\begin{array}{l}\text { Response rate } \\
\text { a }\end{array}$ & $6(15)$ & $8(27)$ \\
$\begin{array}{l}\text { Safety population (n) } \\
\text { Any grade 3 AE }\end{array}$ & 40 & $31(78)$ \\
$\begin{array}{l}\text { Any grade 4 AE } \\
\text { Dose modification }\end{array}$ & $19(48)$ & $24(77)$ \\
$\begin{array}{l}\text { Discontinuation } \\
\text { due to AE }\end{array}$ & $8(20)$ & $11(35)$ \\
\hline
\end{tabular}

$\mathrm{AE}$ adverse event

${ }^{\text {a }}$ Confirmed or unconfirmed best response of complete response or partial response

expansion cohort (ixazomib dose: $2.97 \mathrm{mg} / \mathrm{m}^{2}$ ) in the study of weekly ixazomib. Key efficacy and safety data from these studies are summarized in Table 1 . The overall response rate was numerically higher, and rates of grade 4 AEs, dose modifications, and discontinuations due to AEs were numerically lower, in the weekly versus twice-weekly study. These differences were not compared statistically but were regarded as clinically significant, thereby resulting in a subsequent focus on the weekly dosing regimen in the TOURMALINE clinical trial program.

In the phase I/II study of IRd, evaluating weekly ixazomib dosing, 65 patients with newly diagnosed MM were enrolled and treated at ixazomib doses of $1.68-3.95 \mathrm{mg} / \mathrm{m}^{2}$, with six patients receiving the MTD of $2.97 \mathrm{mg} / \mathrm{m}^{2}$ [14]. In phase I, one dose-limiting toxicity, of grade 3 urticarial rash, was seen in one of the first three patients treated at the MTD, and responses included one complete response (CR), one very good partial response (VGPR), and four partial responses (PRs) at $2.97 \mathrm{mg} / \mathrm{m}^{2}$. At the next lower dose level, $2.23 \mathrm{mg} / \mathrm{m}^{2}$, there were no dose-limiting toxicities among the three patients treated at this dose, and responses included two stringent CRs and one VGPR. Treatment exposure data showed that the median RDI of lenalidomide (defined as the dose received divided by

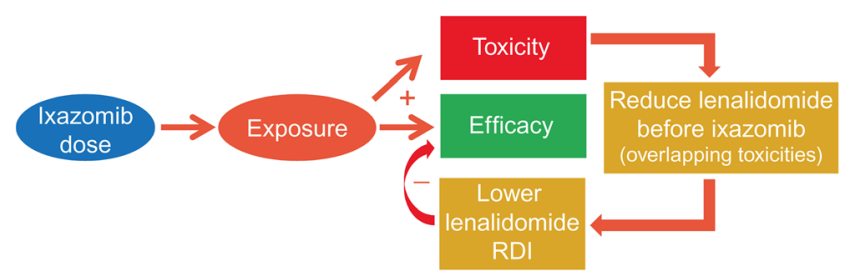

Fig. 1 Schematic illustrating the relationship between ixazomib dose, systemic exposure and the relative dose intensity of lenalidomide in the ixazomib plus lenalidomide and dexamethasone (IRd) regimen. RDI relative dose intensity 
the dose prescribed, as a percentage) was $96 \%$ and $84.6 \%$ in patients who received ixazomib 2.23 and $2.97 \mathrm{mg} / \mathrm{m}^{2}$, respectively. The RDI of lenalidomide was therefore impacted by the higher dose of ixazomib as a result of overlapping toxicities (Fig. 1). Based on this review of safety and efficacy information, the recommended phase II dose was selected as $2.23 \mathrm{mg} /$ $\mathrm{m}^{2}$, which was converted to a fixed dose of $4.0 \mathrm{mg}$ based on an early-phase population pharmacokinetic analysis demonstrating the lack of an effect of BSA or body weight on ixazomib clearance [11]. This dose and regimen was subsequently tested in the phase III TOURMALINE-MM1 study.

\subsection{Exposure-Response Analyses of the Phase III Study of Ixazomib}

\subsubsection{Exposure-Progression-Free Survival Relationship}

In the ITT analysis of TOURMALINE-MM1, PFS was superior with IRd $(n=360)$ versus placebo-Rd $(n=362)$ (hazard ratio [HR] 0.74, median PFS 20.6 vs. 14.7 months, $p=0.012$ ) [4]. For the analysis of the ixazomib exposure-PFS relationship, the 342 evaluable patients were stratified by four ixazomib exposure quartiles and PFS was analyzed and compared with the placebo-Rd regimen (Fig. 2; Table 2). Median PFS was longer in all ixazomib exposure quartiles in the IRd arm than in the placebo-Rd arm, and all HRs were $<1$. Using the Cox proportional hazards model, ixazomib exposure was not a significant predictor of PFS (HR 1.002; 95\% confidence interval $[\mathrm{CI}] 0.998-1.006 ; p=0.2569)$. Thus, no additional covariate analysis was conducted.

\subsubsection{Exposure-Clinical Response Analysis}

In the ITT analysis, the overall response rate was significantly higher with IRd than with placebo-Rd (78\% vs. $72 \%$; $p=0.04$ ), including $12 \%$ versus $7 \%$ with $\mathrm{CR}$ and $48 \%$ versus $39 \%$ with VGPR or better ( $\geq$ VGPR) [4]. Response rates in the four ixazomib exposure quartiles are summarized in Table 2. In the exposure-clinical response analysis (Fig. 3), ixazomib exposure was not a statistically significant predictor of the probability of CR (odds ratio [OR] 1.006; 95\% CI 0.999 $1.012 ; p=0.0778$ ), $\geq$ VGPR (OR $1.003 ; 95 \%$ CI 0.998 $1.008 ; p=0.2041)$, or PR or better $(\geq \mathrm{PR})(\mathrm{OR} 0.995 ; 95 \%$ CI $0.989-1.001 ; p=0.0770)$, based on logistic regression analysis.

\subsubsection{Exposure-Safety Analysis}

Rates of selected grade $\geq 3$ hematologic AEs (anemia, neutropenia, thrombocytopenia) and grade $\geq 2$ non-hematologic AEs (diarrhea, fatigue, nausea, peripheral neuropathy, rash) ascribed to ixazomib were evaluated in the four ixazomib exposure quartiles in the IRd arm (Table 3).

Using logistic regression modeling, statistically significant associations were observed between ixazomib exposure and the probability of grade $\geq 3$ anemia (OR 1.007 ; 95\% CI 1.002 $1.013 ; p=0.0117$; Fig. $4 \mathrm{a}$ ) and grade $\geq 3$ thrombocytopenia (OR 1.013; 95\% CI 1.008-1.018; $p<0.0001$; Fig. 4c), but not grade $\geq 3$ neutropenia (OR $1.000 ; 95 \%$ CI $0.995-1.006$; $p=0.8734$; Fig. $4 \mathrm{~b})$. For anemia and thrombocytopenia, none of the tested covariates had a significant effect on the

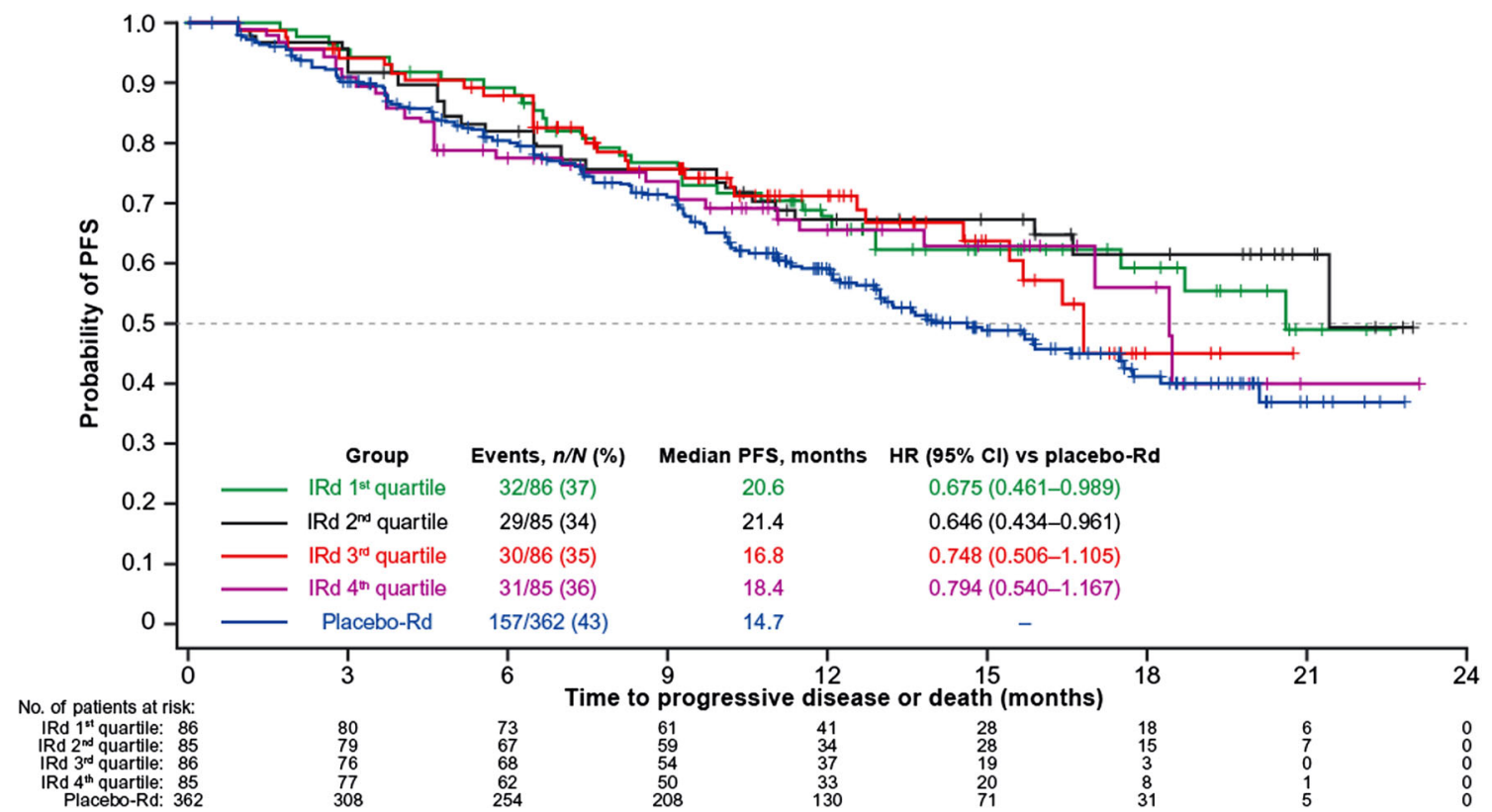

Fig. 2 Ixazomib exposure-progression-free survival (PFS) analysis at the $\sim 15$-month analysis of TOURMALINE-MM1 in the ixazomib plus lenalidomide and dexamethasone (IRd) and placebo plus lenalidomide and dexamethasone (placebo-Rd) arms. Kaplan-Meier curves show PFS distributions in the placebo-Rd arm and in the IRd arm by quartiles of ixazomib exposure. CI confidence interval, HR hazard ratio 
exposure-AE relationships and therefore were not included in the final model.

Based on prior pharmacokinetic data [20] showing a multiple-dose ixazomib $\mathrm{AUC}_{0-168 \mathrm{~h}}$ of $990 \mathrm{ng} \cdot \mathrm{h} / \mathrm{mL}$ after weekly administration of ixazomib $4.0 \mathrm{mg}$, the timeaveraged ixazomib exposure for a $4.0 \mathrm{mg}$ dose is $\sim 141 \mathrm{ng} \cdot \mathrm{h} /$ $\mathrm{mL} /$ day (i.e., $990 \mathrm{ng} \cdot \mathrm{h} / \mathrm{mL}$ divided by 7 days). Similarly, a $1.0 \mathrm{mg}$ dose is equivalent to an ixazomib exposure of $\sim 35 \mathrm{ng} \cdot \mathrm{h} / \mathrm{mL} /$ day. Because ixazomib exhibits dose-linear pharmacokinetics $[10,13]$, the corresponding time-averaged ixazomib exposure for a $3.0 \mathrm{mg}$ dose (representing first dose reduction level) is estimated as $\sim 106 \mathrm{ng} \cdot \mathrm{h} / \mathrm{mL} /$ day, which represents a difference of $\sim 35 \mathrm{ng} \cdot \mathrm{h} / \mathrm{mL} /$ day compared with the $4.0 \mathrm{mg}$ dose. Of note, this difference in time-averaged ixazomib exposure between a 4.0 and a $3.0 \mathrm{mg}$ dose is similar to the difference in median ixazomib exposure between the second and third exposure quartiles in these analyses. Based on these data, an increase in ixazomib exposure of $35 \mathrm{ng} \cdot \mathrm{h} /$ $\mathrm{mL}$ /day was predicted to be associated with $28 \%$ (OR 1.278) and 58\% (OR 1.576) increases in the odds of grade $\geq 3$ anemia and thrombocytopenia, respectively. Conversely, a decrease in ixazomib exposure of $35 \mathrm{ng} \cdot \mathrm{h} / \mathrm{mL} /$ day was predicted to be associated with $22 \%$ (OR 0.783 ) and $37 \%$ (OR 0.634) decreases in the respective odds of these hematologic AEs.

Using logistic regression modeling for the nonhematologic AEs, statistically significant associations were observed between ixazomib exposure and the probability of grade $\geq 2$ diarrhea (OR 1.012; 95\% CI 1.007-1.017; $p<0.0001$ ), grade $\geq 2$ fatigue (OR 1.006; 95\% CI 1.000 $1.012 ; p=0.0358$; with age as a statistically significant covariate, OR 1.055; 95\% CI 1.014-1.098; $p=0.0088$ ), grade $\geq 2$ nausea (OR 1.012; 95\% CI 1.004-1.019; $p=0.0019$ ), grade $\geq 2$ peripheral neuropathy (OR 1.006; 95\% CI 1.000-1.012; $p=0.0495)$, and grade $\geq 2$ rash (OR 1.020; 95\% CI 1.014 1.026; $p<0.0001$ ) (Fig. 4d-h). For diarrhea, nausea, peripheral neuropathy, and rash, none of the covariates tested had a significant effect on the exposure-AE relationship and therefore were not included in the final model. Based on the final logistic regression models for each $\mathrm{AE}$, an increase in ixazomib exposure of $35 \mathrm{ng} \cdot \mathrm{h} / \mathrm{mL} /$ day was predicted to be associated with 52\% (OR 1.522), 23\% (OR 1.234), 52\% (OR 1.522), 23\% (OR 1.234), and 101\% (OR 2.014) increases in the odds of grade $\geq 2$ diarrhea, fatigue, nausea, peripheral neuropathy, and rash, respectively. Conversely, a decrease in ixazomib exposure of $35 \mathrm{ng} \cdot \mathrm{h} / \mathrm{mL} /$ day was predicted to be associated with $34 \%$ (OR 0.657), 19\% (OR 0.811), 34\% (OR 0.657), 19\% (OR 0.811), and 50\% (OR 0.497) decreases in the odds of these grade $\geq 2$ AEs, respectively.

\subsubsection{Exposure-Time to Dose Reduction Analysis}

Figure 5 shows the relationship between ixazomib exposure quartile and time to first ixazomib dose reduction by 

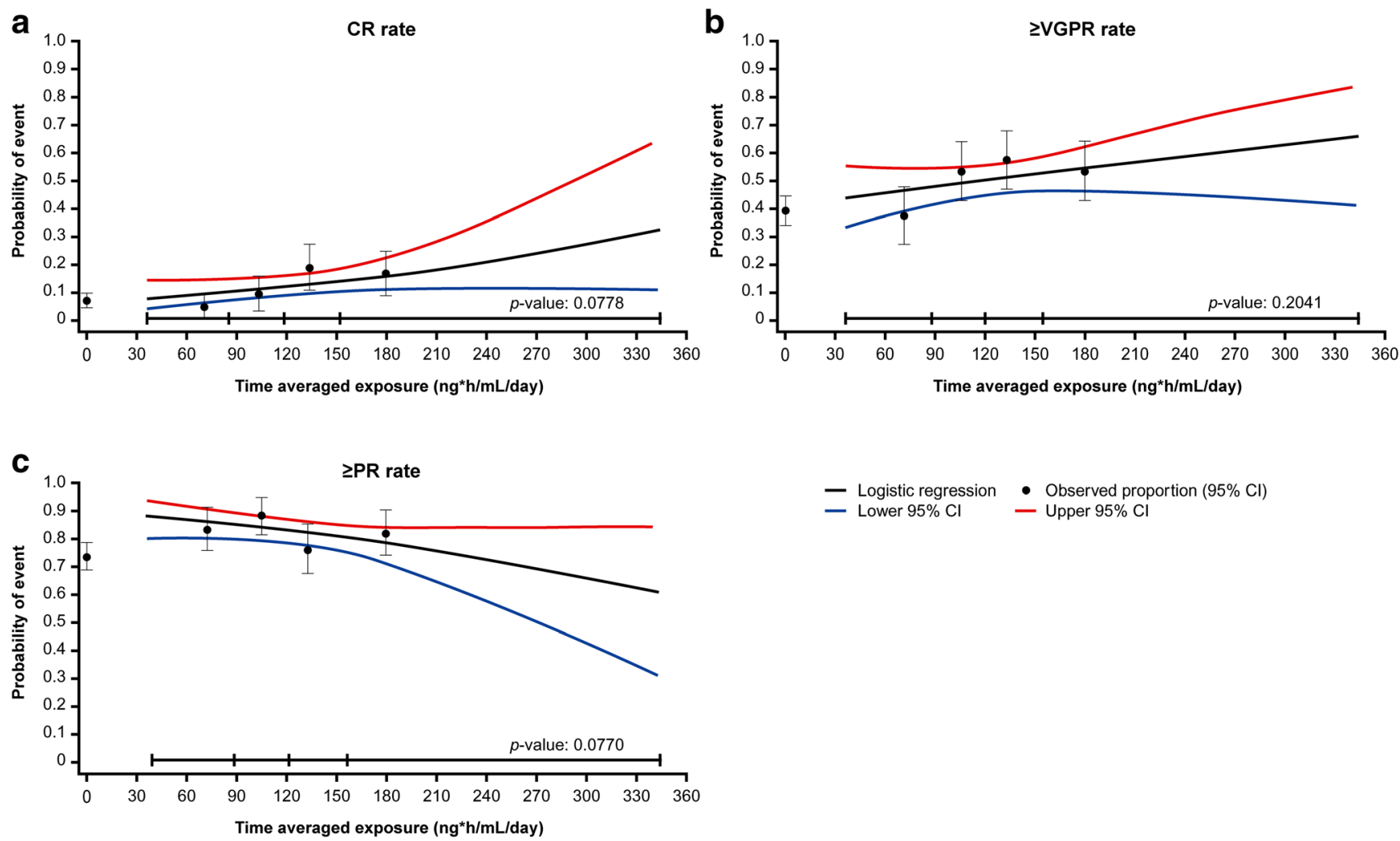

Fig. 3 Ixazomib exposure-response analysis at the $\sim 15$-month analysis of TOURMALINE-MM1 in the ixazomib plus lenalidomide and dexamethasone (IRd) and placebo plus lenalidomide and dexamethasone (placebo-Rd) arms. Observed incidence and predicted probability of (a) complete response (CR), (b) very good partial response or better

( $\geq$ VGPR), and (c) partial response or better ( $\geq P R$ ), as a function of ixazomib exposure using a logistic regression model. Black circles and error bars show the response probabilities plus $95 \%$ confidence interval (CI) in the placebo-Rd arm and in the IRd arm within each ixazomib exposure quartile (left to right); data summarized in Table 2

Kaplan-Meier analysis, along with time to first placebo dose reduction in the placebo-Rd arm. Results from the Cox proportional hazards model indicated that ixazomib exposure was not a predictor of time to first ixazomib dose reduction (HR 1.004; 95\% CI 1.000-1.009; $p=0.0693)$. The number of ixazomib dose reductions was small. After a median of 12 cycles, only $20 \%$ of patients in the IRd arm required one or more ixazomib dose reduction, indicating that $80 \%$ of patients were able to tolerate the $4.0 \mathrm{mg}$ starting dose of ixazomib.

\subsubsection{Exposure-Lenalidomide Relative Dose Intensity Analysis}

Figure 6 shows the relationship between ixazomib exposure quartile, based on $\mathrm{AUC}_{\mathrm{inf}}$, and the proportion of patients with a lenalidomide RDI of $\geq 60 \%$, which represents a one-level lenalidomide dose reduction. Higher ixazomib exposures were associated with a lower probability of lenalidomide RDI being $\geq 60 \%$. The relationship was significant $(p<0.0001)$, and similar relationships were observed for other cut-offs of lenalidomide RDI, including $70 \%$ and $80 \%$ (data not shown). It should be noted that these observations are

made within the constraints of the protocol-specified dosemodification criteria in the TOURMALINE-MM1 study, in which lenalidomide dose was reduced before ixazomib dose for specific toxicities (e.g., thrombocytopenia [platelets $<30 \times 10^{9} / \mathrm{L}$ ], neutropenia [absolute neutrophil count $\left.<0.5 \times 10^{9} / \mathrm{L}\right]$, grade $2 / 3$ rash [21]).

\section{Discussion}

The findings of the analyses reported herein provide the rationale and justification for the dose and schedule of ixazomib used in the pivotal, global, randomized, double-blind, placebo-controlled phase III TOURMALINE-MM1 study in relapsed/refractory MM [4], and in the ongoing TOURMALINE-MM2 study in newly diagnosed MM (NCT01850524), as well as multiple investigator-initiated studies. This dose and schedule of ixazomib $4.0 \mathrm{mg}$ on days 1,8 , and 15 of a 28 -day cycle, in combination with lenalidomide $25 \mathrm{mg}$ on days $1-21$ and dexamethasone $40 \mathrm{mg}$ on days $1,8,15$, and 22 , is the recommended starting dose for patients with relapsed/refractory MM $[2,3]$. These findings also provide insights to address the question of 
Table 3 Summary of rates of selected grade $\geq 3$ hematologic adverse events (AEs) and grade $\geq 2$ non-hematologic AEs in the four ixazomib exposure quartiles of the ixazomib plus lenalidomide and dexamethasone (IRd) arm, compared with the placebo plus lenalidomide and dexamethasone (placeboRd) arm

\begin{tabular}{|c|c|c|c|c|c|c|}
\hline & \multicolumn{5}{|l|}{ IRd arm } & \multirow{2}{*}{$\begin{array}{l}\text { Placebo-Rd } \\
(n=360)\end{array}$} \\
\hline & $\begin{array}{l}\text { Total } \\
(n=347)\end{array}$ & $\begin{array}{l}\text { 1st quartile } \\
(n=87)\end{array}$ & $\begin{array}{l}\text { 2nd quartile } \\
(n=87)\end{array}$ & $\begin{array}{l}\text { 3rd quartile } \\
(n=87)\end{array}$ & $\begin{array}{l}\text { 4th quartile } \\
(n=86)\end{array}$ & \\
\hline \multicolumn{7}{|l|}{ Hematologic AEs } \\
\hline Anemia, $n(\%)$ & $31(9)$ & $7(8)$ & $5(6)$ & $7(8)$ & $12(14)$ & $45(13)$ \\
\hline $\begin{array}{l}\text { Median exposure, } \\
\text { ng.h/mL/day (range) }\end{array}$ & $120(34.9-641)$ & $72.1(34.9-86.2)$ & $105(87.0-120)$ & $134(122-155)$ & $186(156-641)$ & NA \\
\hline Neutropenia, $n(\%)$ & $69(20)$ & $16(18)$ & $18(21)$ & $19(22)$ & $16(19)$ & $71(20)$ \\
\hline $\begin{array}{l}\text { Median exposure, } \\
\text { ng.h/mL/day (range) }\end{array}$ & $123(34.9-357)$ & $72.1(34.9-87.5)$ & $109(87.7-123)$ & $138(123-157)$ & $186(157-357)$ & NA \\
\hline Thrombocytopenia, $n(\%)$ & $59(17)$ & $7(8)$ & $10(11)$ & $14(16)$ & $28(33)$ & $26(7)$ \\
\hline $\begin{array}{l}\text { Median exposure, } \\
\mathrm{ng} \cdot \mathrm{h} / \mathrm{mL} / \text { day (range) }\end{array}$ & $123(34.9-516)$ & $70.7(34.9-87.1)$ & $107(87.3-123)$ & $139(123-158)$ & $195(159-516)$ & NA \\
\hline \multicolumn{7}{|l|}{ Non-hematologic AEs } \\
\hline Diarrhea, $n(\%)$ & $70(20)$ & $9(10)$ & $15(17)$ & $12(14)$ & $34(40)$ & $53(15)$ \\
\hline $\begin{array}{l}\text { Median exposure, } \\
\text { ng.h/mL/day (range) }\end{array}$ & $123(34.9-420)$ & $72.5(34.9-88.8)$ & $107(89.1-123)$ & $139(124-161)$ & $195(162-420)$ & NA \\
\hline Fatigue, $n(\%)$ & $40(12)$ & $11(13)$ & $6(7)$ & $8(9)$ & $15(17)$ & $49(14)$ \\
\hline $\begin{array}{l}\text { Median exposure, } \\
\text { ng.h/mL/day (range) }\end{array}$ & $122(36.6-430)$ & $72.1(36.6-87.1)$ & $106(87.3-122)$ & $138(122-157)$ & $191(157-430)$ & NA \\
\hline Nausea, $n(\%)$ & $21(6)$ & $2(2)$ & $4(5)$ & $5(6)$ & $10(12)$ & $24(7)$ \\
\hline $\begin{array}{l}\text { Median exposure, } \\
\text { ng.h/mL/day (range) }\end{array}$ & $122(34.9-344)$ & $72.1(34.9-87.0)$ & $105(87.1-122)$ & $137(123-156)$ & $185(156-344)$ & NA \\
\hline Peripheral neuropathy, n (\%) & $35(10)$ & $8(9)$ & $6(7)$ & $9(10)$ & $12(14)$ & $26(7)$ \\
\hline $\begin{array}{l}\text { Median exposure, } \\
\text { ng.h/mL/day (range) }\end{array}$ & $120(34.9-444)$ & $70.3(34.9-87.1)$ & $105(87.1-120)$ & $137(120-155)$ & $185(155-344)$ & NA \\
\hline Rash, n (\%) & $49(14)$ & $2(2)$ & $9(10)$ & $5(6)$ & $33(38)$ & $25(7)$ \\
\hline $\begin{array}{l}\text { Median exposure, } \\
\text { ng.h/mL/day (range) }\end{array}$ & $124(29.1-679)$ & $72.5(29.1-88.7)$ & $107(89.3-124)$ & $145(125-167)$ & $214(168-679)$ & NA \\
\hline
\end{tabular}

NA not applicable

whether, given the tolerability demonstrated with IRd in TOURMALINE-MM1 and the limited additional and manageable toxicity reported with IRd versus placebo-Rd [4], a higher dose of ixazomib or a more intensive administration schedule might be a feasible consideration for IRd.

Evaluation of findings from the early-phase studies of single-agent ixazomib $[13,18]$ highlighted the apparently favorable efficacy/safety profile associated with weekly versus twice-weekly dosing at the respective MTDs. Data from a subsequent phase I/II investigation of weekly ixazomib in combination with Rd identified an ixazomib dose that provided an acceptable efficacy/safety profile without negatively impacting lenalidomide RDI [14]. These phase I/II findings also suggested that a weekly dose of ixazomib higher than $4.0 \mathrm{mg}$ would be associated with a poorer safety profile. Furthermore, due to overlapping toxicities with lenalidomide, such as thrombocytopenia, gastrointestinal toxicities, and rash $[4,13,14,18,22]$, higher doses of ixazomib would be expected to adversely affect the lenalidomide RDI.
Importantly, the phase I/II findings were reflected in the outcomes of the exposure-response analyses conducted using data from the TOURMALINE-MM1 phase III study. The key findings of these analyses were that ixazomib exposure was not a predictor for clinical efficacy in terms of PFS or response with IRd in patients with relapsed/refractory MM, but that there were statistically significant associations between ixazomib exposure and the risk of specific AEs, as well as the RDI of lenalidomide. The exposure-PFS/response analyses suggested that the efficacy benefit of IRd was consistent across the range of exposures achieved using the weekly $4.0 \mathrm{mg}$ dose of ixazomib. One potential explanation of the lack of an apparent exposure-efficacy relationship is a greater variability in intrinsic sensitivity to treatment response within the patient population due to other prognostic and patient-specific disease factors as compared to variability in ixazomib systemic exposure. Another possible explanation is associated with the impact of ixazomib systemic exposure on lenalidomide RDI (Fig. 6). Specifically, higher ixazomib exposure may potentially have 
- Logistic regression - Observed proportion $(95 \% \mathrm{Cl})$ - Lower $95 \% \mathrm{Cl} \quad$ - Upper $95 \% \mathrm{Cl}$

a

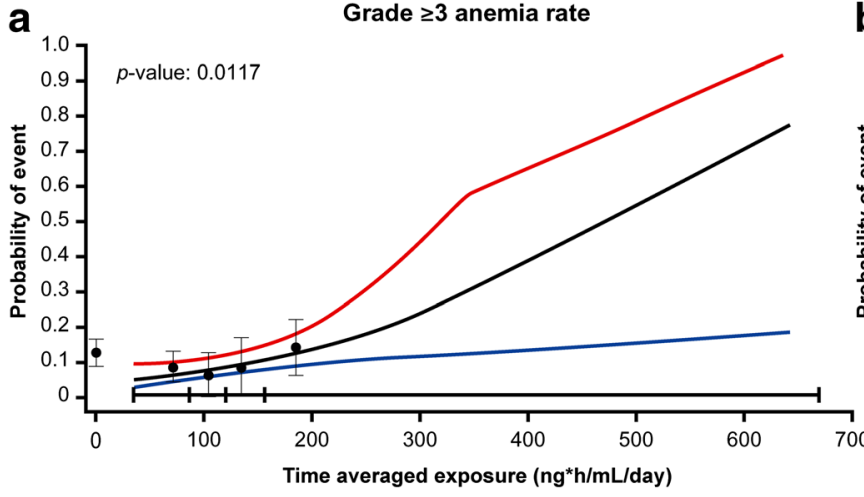

C

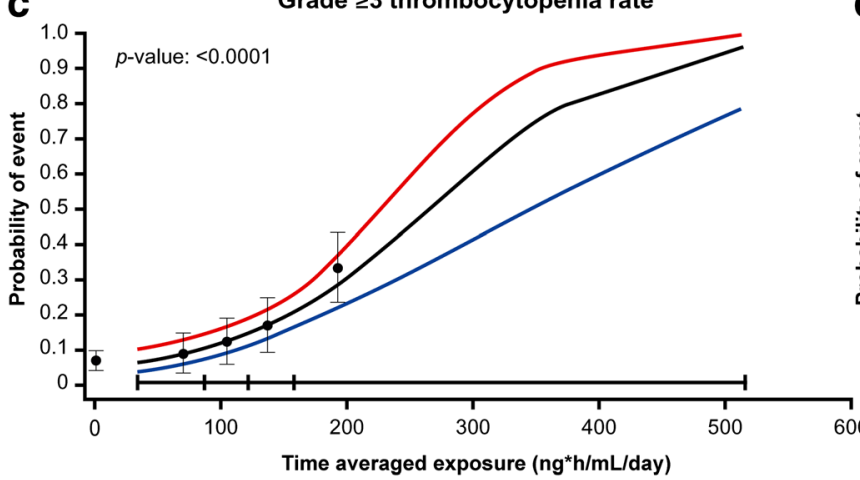

e

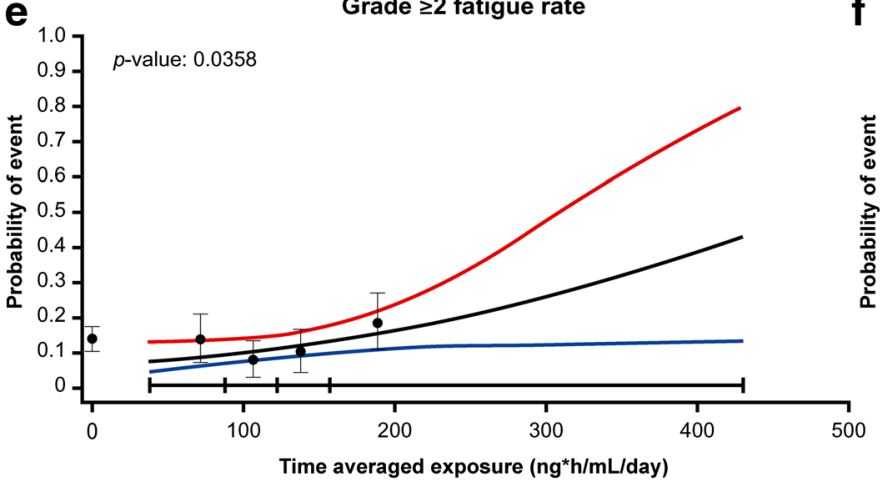

g

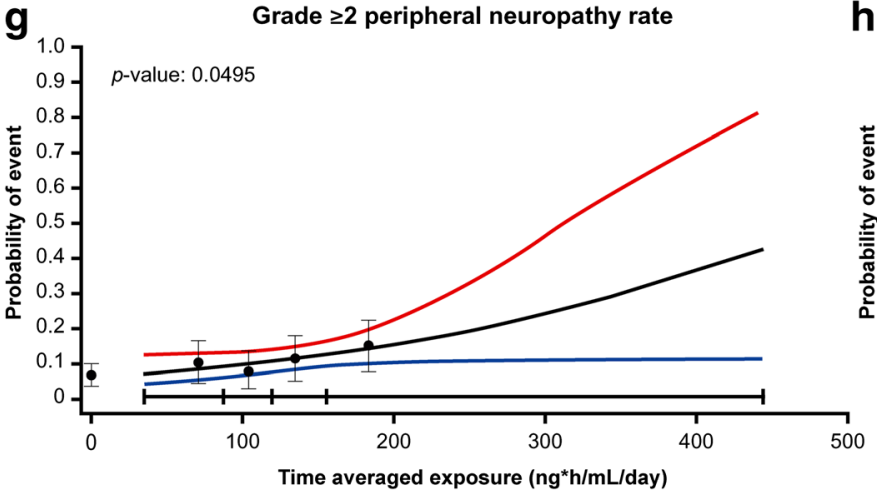

d

$\mathbf{f}$
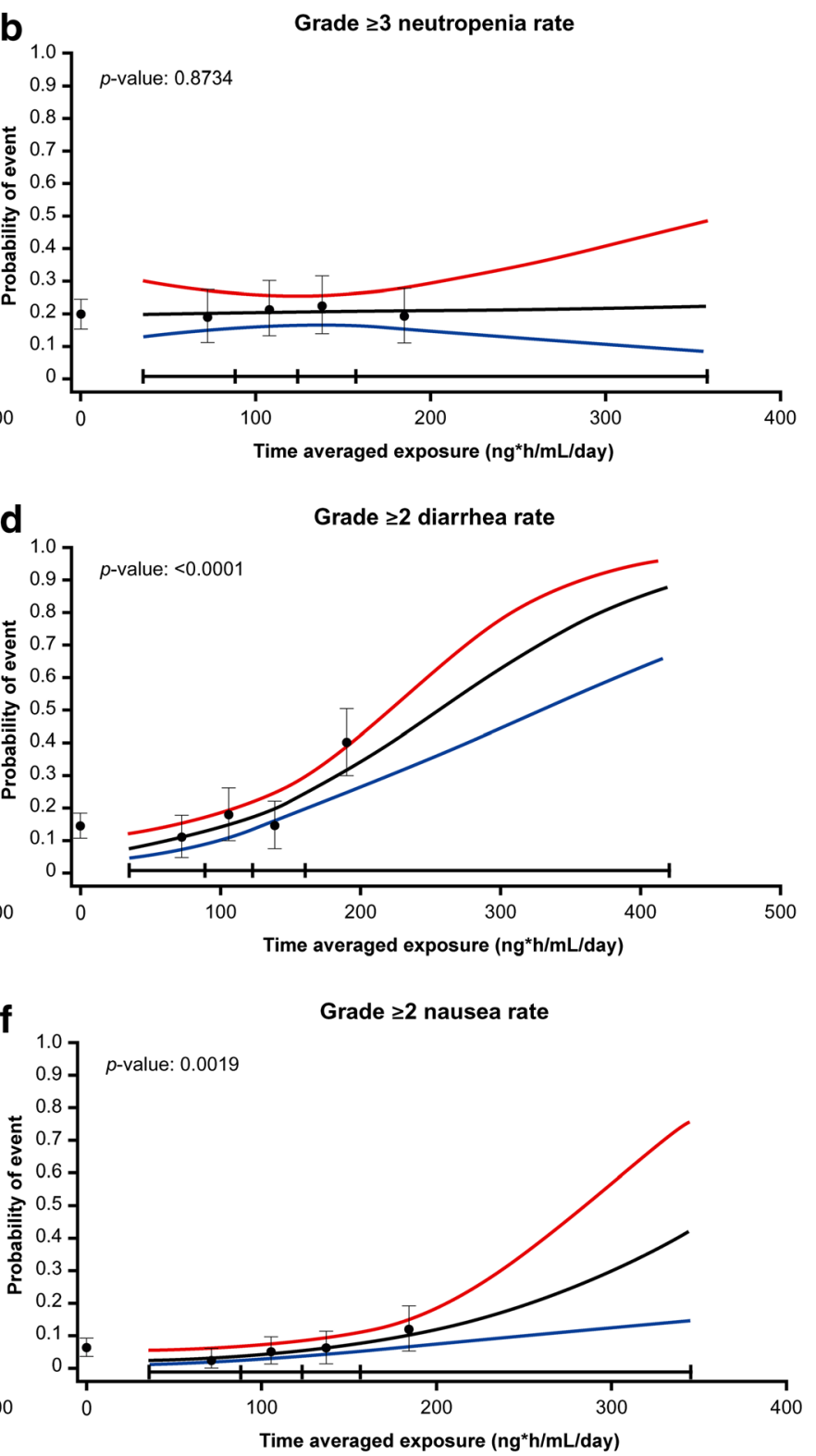

h

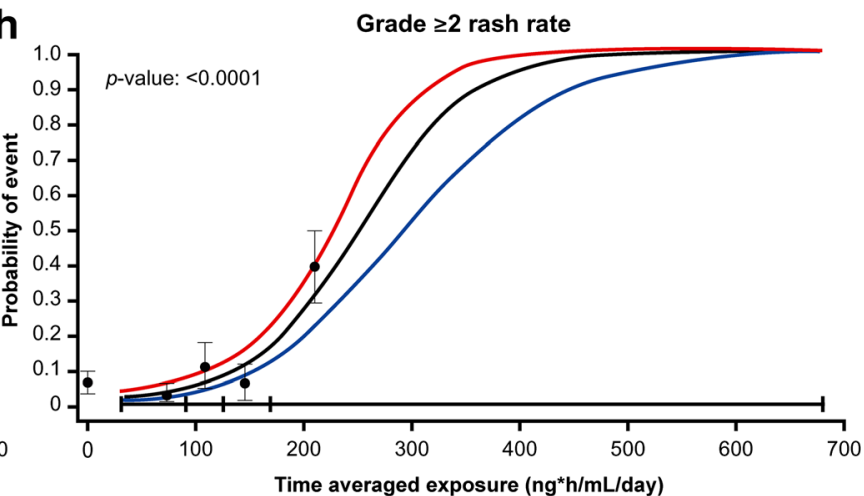


4 Fig. 4 Observed incidence and predicted probability of (a) grade $\geq 3$ anemia, (b) grade $\geq 3$ neutropenia, (c) grade $\geq 3$ thrombocytopenia, (d) grade $\geq 2$ diarrhea, (e) grade $\geq 2$ fatigue, (f) grade $\geq 2$ nausea, (g) grade $\geq 2$ peripheral neuropathy, and (h) grade $\geq 2$ rash as a function of ixazomib exposure using a logistic regression model. Black circles and error bars show the event probabilities plus $95 \%$ confidence interval (CI) in the placebo plus lenalidomide and dexamethasone (placebo-Rd) arm and in the ixazomib plus lenalidomide and dexamethasone (IRd) arm within each ixazomib exposure quartile (left to right); data summarized in Table 3

led to improved PFS and response with IRd if lenalidomide RDI could have been maintained, for example through using different dose-modification criteria in TOURMALINE-MM1 to allow patients to tolerate more toxicity. However, as lenalidomide RDI was reduced with greater ixazomib systemic exposure, the former may have counterbalanced the latter in terms of potential impact on efficacy (Fig. 7), resulting in no ixazomib exposure-PFS/response relationship. The overlapping mechanisms of action of proteasome inhibitors and immunomodulatory drugs, including nuclear factor-kappa B inhibition, caspase-mediated apoptosis, and inhibition of myeloma cell migration and angiogenesis [23-27], have been shown to have synergistic activity in myeloma [26-29], with proteasome inhibitors plus Rd having demonstrated high response rates and longer PFS in multiple phase III studies [4, 30, 31]. The specific mechanisms resulting in proteasome inhibitor-immunomodulatory drug synergy remain to be elucidated, and, to date, studies have not explored the importance of the sequencing of the agents in contributing to the synergistic effect.

Exposure-safety analyses demonstrated a relationship between ixazomib exposure and several AEs. For grade $\geq 3$ anemia, this relationship was primarily driven by the incidence seen in the fourth quartile of ixazomib exposure (14\%), which was similar to that observed with placebo-Rd (13\%) [4]; the incidence in the other three quartiles of ixazomib exposure was lower (6-8\%). Given these findings, the exposure-anemia relationship was inferred to not be of clinical relevance at the doses administered in TOURMALINE-MM1. Similarly, a significant relationship was seen between exposure and grade $\geq 3$ thrombocytopenia. This finding is consistent with the pharmacological mechanism of action of ixazomib, as proteasome inhibition causes transient inhibition of platelet budding from megakaryocytes [32, 33]. However, rates of serious AEs of thrombocytopenia ( $2 \%$ and $2 \%$ ) and the need for platelet transfusions ( $8 \%$ and $6 \%$ ) were similar between the IRd and placeboRd arms, respectively, in TOURMALINE-MM1 [4]; thus, the significant exposure-thrombocytopenia relationship was not considered to be of clinical relevance due to the lack of increased risk of sequelae in the IRd arm. For non-hematologic AEs, significant relationships were seen between ixazomib exposure and grade $\geq 2$ rash, peripheral neuropathy, fatigue, nausea, and diarrhea. However, in TOURMALINE-MM1, these AEs were readily manageable, and were not associated with major clinical complications or substantial rates of discontinuations $[4,21]$.

The relationships determined in these exposure-safety analyses provide support for the dose reduction of ixazomib from 4.0 to $3.0 \mathrm{mg}$ in patients experiencing intolerable AEs as recommended in the dose-modification guidelines section of the ixazomib prescribing information $[2,3]$. The difference in time-averaged ixazomib exposure between a 4.0 and a $3.0 \mathrm{mg}$ dose was estimated to be $\sim 35 \mathrm{ng} \cdot \mathrm{h} / \mathrm{mL} /$ day, and logistic

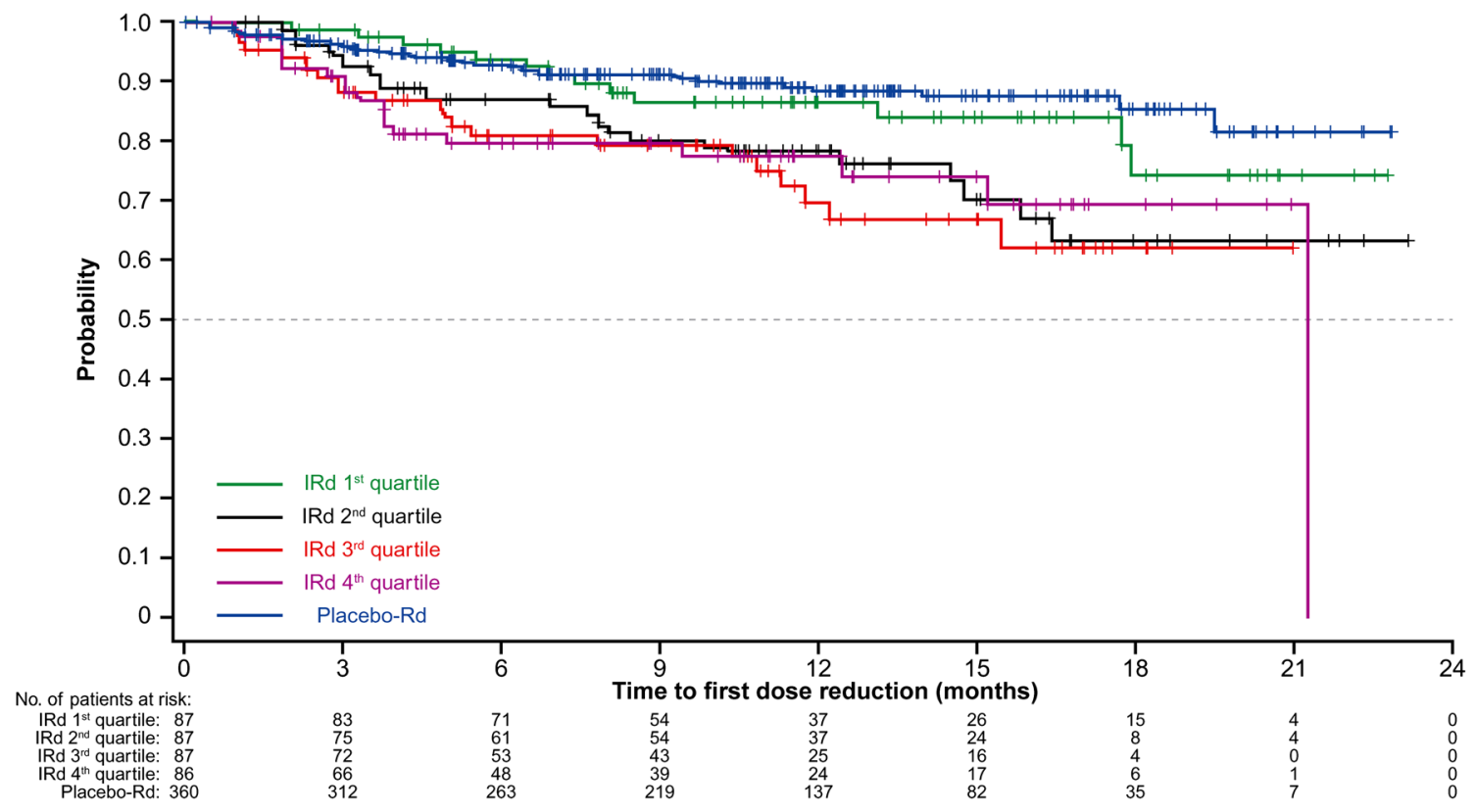

Fig. 5 Kaplan-Meier estimates for time to first ixazomib dose reduction in the four time-averaged ixazomib exposure quartiles in the ixazomib plus lenalidomide and dexamethasone (IRd) arm of TOURMALINE-

MM1, compared with time to first placebo dose reduction in the placebo plus lenalidomide and dexamethasone (placebo-Rd) arm 


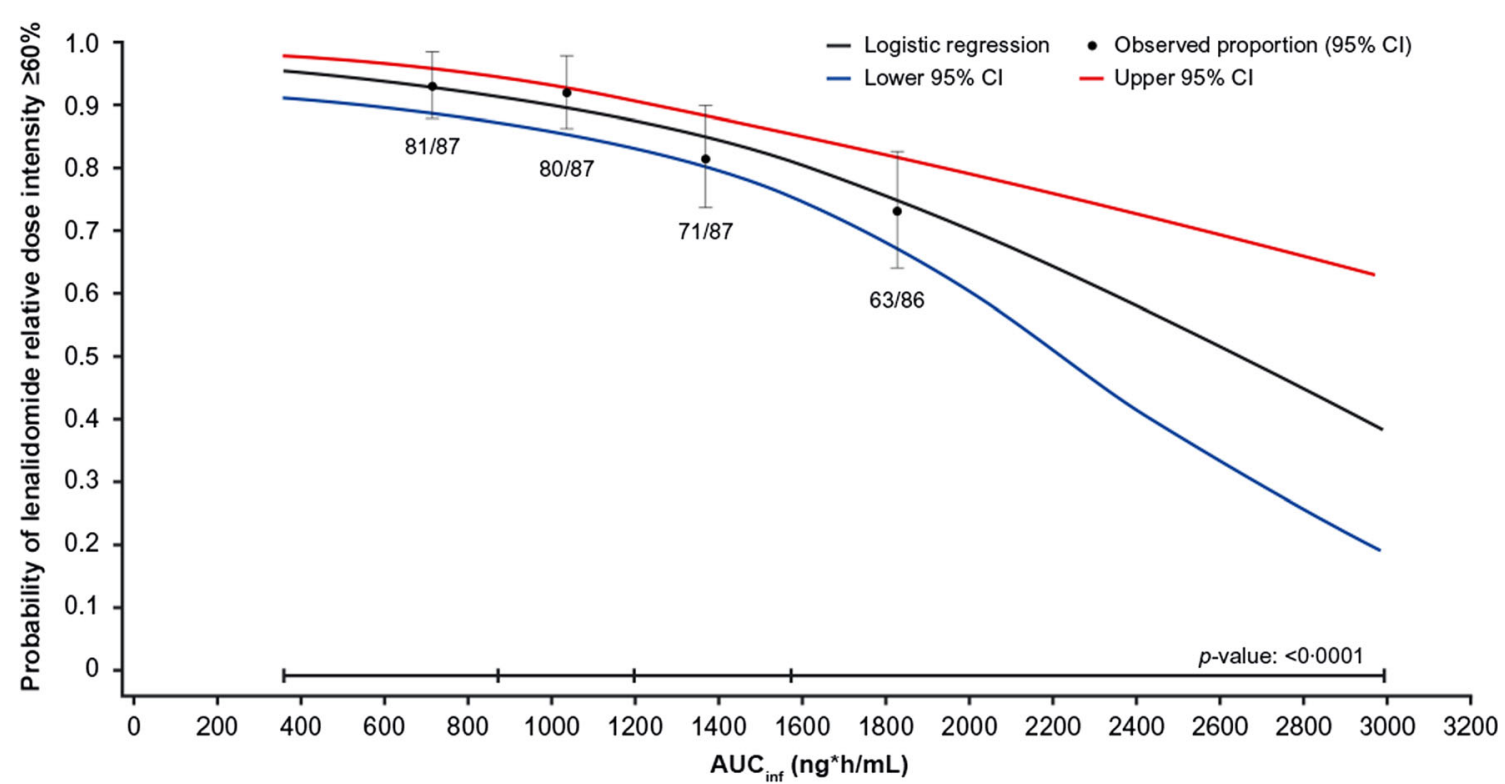

Fig. 6 Analysis of lenalidomide relative dose intensity according to ixazomib exposure $\left(\mathrm{AUC}_{\mathrm{inf}}\right)$ in the ixazomib plus lenalidomide and dexamethasone (IRd) arm of TOURMALINE-MM1, showing probability of lenalidomide relative dose intensity $\geq 60 \%$. Black circles and error bars show the event probabilities plus $95 \%$ confidence interval (CI) in the IRd arm within each ixazomib exposure quartile regression modeling demonstrated that a decrease in ixazomib exposure of this magnitude was predicted to be associated with decreases in the odds of grade $\geq 3$ hematologic AEs or grade $\geq 2$ non-hematologic AEs of between 19\% and 50\%. Similarly, these findings support the recommended reduced starting dose of $3.0 \mathrm{mg}$ of ixazomib in patients with moderate-to-severe hepatic impairment [34] and in patients with severe renal impairment or end-stage renal disease requiring dialysis [35], in whom ixazomib exposure was shown to be increased as compared to patients with normal organ function.

IRd was shown to be well-tolerated in TOURMALINEMM1, with high and similar median RDIs for both ixazomib/placebo $(97.4 \% / 98.8 \%)$, lenalidomide $(93.8 \% /$ $96.6 \%)$ and dexamethasone $(92.2 \% / 94.9 \%)$ in the IRd/ placebo-Rd arms overall. Additionally, a limited rate of

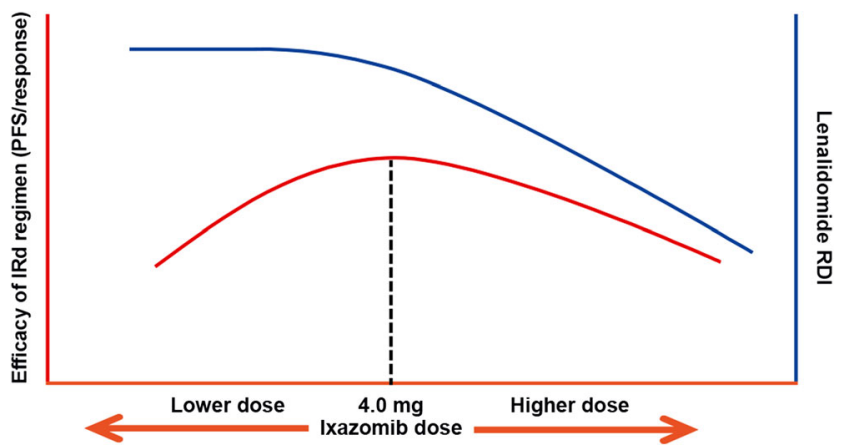

Fig. 7 Proposed framework illustrating the impact of ixazomib dose on the relative dose intensity (RDI) of lenalidomide (top, blue curve) and on the efficacy of the ixazomib plus lenalidomide and dexamethasone (IRd) regimen (bottom, orange curve). Note, this visual representation is not intended to reflect a quantitative estimation of the dose-response relationship ixazomib dose reductions, and low and similar rates of discontinuations due to AEs (17\%/14\%), were observed in the IRd/placebo-Rd arms [4]. These clinical observations are supported by the exposure-ixazomib dose reduction analysis, which showed no significant relationship between ixazomib exposure and time to first ixazomib dose reduction (Fig. 5).

In conclusion, evaluation of early-phase study data and the exposure-response analyses of TOURMALINE-MM1 data support a favorable benefit-risk profile for the ixazomib dose and schedule used in TOURMALINE-MM1 and TOURMALINE-MM2, which is also the dosing regimen approved by regulatory authorities for patients with relapsed/ refractory MM $[2,3]$. The findings of the exposure-response analyses and the phase I/II study suggest that ixazomib doses $>4.0 \mathrm{mg}$, such as the MTD of $5.5 \mathrm{mg}$, in combination with Rd, may lead to higher rates of AEs and/or more severe AEs. Moreover, higher doses of ixazomib will result in increased systemic exposures, which are anticipated to negatively impact lenalidomide RDI; this may potentially counterbalance the possible positive effects of a higher ixazomib dose on the overall efficacy of IRd (Fig. 7). However, the findings of these analyses of TOURMALINE-MM1 data should be interpreted within the constraints of the protocol-specified dose-modification criteria in which lenalidomide dose was reduced before ixazomib dose for specific toxicities. Additionally, these analyses suggest that ixazomib dose titration (e.g., starting at $4.0 \mathrm{mg}$ and escalating to $5.5 \mathrm{mg}$ ) might be feasible for a subgroup of patients in whom the $4.0 \mathrm{mg}$ dose is tolerable or in clinical situations in which greater toxicity associated with a higher dose may be more acceptable to the patient. The success of such an approach may depend upon prophylactically 
managing known AEs in order to limit the need for dose modifications and thus to maintain a high RDI for both ixazomib and lenalidomide, which might translate into further improvements in efficacy. As a related example of the application of context-specific benefit-risk considerations in dose selection, the utility of a titration approach (starting at $3.0 \mathrm{mg}$ and escalating to $4.0 \mathrm{mg}$ ) is being evaluated in the maintenance therapy setting for ixazomib, designed based on a quantitative assessment of exposure-response relationships in the single-agent setting [36]. It has also been suggested that use of twiceweekly administration of ixazomib could be considered for patients who might benefit from more intensive therapy [1], such as relapsed patients who are progressing rapidly, or in the context of induction therapy. However, these alternative approaches would require prospective evaluation to determine their benefit-risk profile relative to the $4.0 \mathrm{mg}$ weekly dose that was studied in TOURMALINE-MM1 and is being used in the TOURMALINE-MM2 study in newly diagnosed MM.

Acknowledgments The authors acknowledge the editorial support of Steve Hill of FireKite, an Ashfield company, part of UDG Healthcare plc, which was funded by Millennium Pharmaceuticals, Inc., and complied with Good Publication Practice 3 ethical guidelines (Battisti WP, et al. Ann Intern Med. 2015;163:461-4).

Authorship Contribution Statements NG was the principal investigator with regards to the exposure-response analyses presented and takes primary responsibility for the paper; SK and PGR collected data and/or recruited patients; NG and KV designed the study analyses. NG, HY, $\mathrm{MJH}, \mathrm{SZ}$, and RL participated in the statistical analysis; NG, HY, MJH, $\mathrm{SZ}$, and RL coordinated preparation and analysis of the dataset; NG, $\mathrm{MJH}$, TS, and KV interpreted the data. NG, MJH, and KV prepared the manuscript. All authors reviewed and approved the manuscript.

\section{Compliance with Ethical Standards}

Funding This study was supported by research funding from Millennium Pharmaceuticals, Inc., Cambridge, MA, USA, a wholly owned subsidiary of Takeda Pharmaceutical Company Limited.

Disclosure of Potential Conflicts of Interest NG, HY, MJH, SZ, RL, $\mathrm{TS}$, and KV are employees of Millennium Pharmaceuticals, Inc., Cambridge, MA, USA, a wholly owned subsidiary of Takeda Pharmaceutical Company Limited.

SK reports research funding from Takeda, Celgene, Novartis, AbbVie, Merck, Janssen, and Sanofi, and honoraria from Skyline, Noxxon, and Kesios.

PGR reports advisory boards for Celgene, Janssen, and Takeda.

Ethical Approval Ethical approval was provided for each of the individual clinical studies from which data were derived for this manuscript.

Informed Consent All patients enrolled to each of the individual clinical studies from which data were derived for this manuscript provided written informed consent.

Open Access This article is distributed under the terms of the Creative Commons Attribution-NonCommercial 4.0 International License (http:// creativecommons.org/licenses/by-nc/4.0/), which permits any noncommercial use, distribution, and reproduction in any medium, provided you give appropriate credit to the original author(s) and the source, provide a link to the Creative Commons license, and indicate if changes were made.

\section{References}

1. Richardson PG, Moreau P, Laubach JP, et al. The investigational proteasome inhibitor ixazomib for the treatment of multiple myeloma. Future Oncol. 2015;11(8):1153-68.

2. Millennium Pharmaceuticals Inc. NINLARO ${ }^{\circledR}$ (ixazomib) capsules, for oral use. Prescribing information, November 2016. http://www.ninlaro.com/downloads/prescribing-information.pdf. Accessed 24 July 2017.

3. Takeda Pharma AS. NINLARO® (ixazomib). Summary of product characteristics. http://www.ema.europa.eu/docs/en_GB/document library/EPAR_-Product_Information/human/003844/ WC500217620.pdf. Accessed 24 July 2017.

4. Moreau P, Masszi T, Grzasko N, et al. Oral Ixazomib, lenalidomide, and dexamethasone for multiple myeloma. N Engl J Med. 2016;374(17):1621-34.

5. Bullock JM, Rahman A, Liu Q. Lessons learned: dose selection of small molecule-targeted oncology drugs. Clin Cancer Res. 2016;22(11):2630-8.

6. Janne PA, Kim G, Shaw AT, et al. Dose finding of small-molecule oncology drugs: optimization throughout the development life cycle. Clin Cancer Res. 2016;22(11):2613-7.

7. Lu D, Lu T, Stroh M, et al. A survey of new oncology drug approvals in the USA from 2010 to 2015: a focus on optimal dose and related postmarketing activities. Cancer Chemother Pharmacol. 2016;77(3):459-76.

8. Sachs JR, Mayawala K, Gadamsetty S, et al. Optimal dosing for targeted therapies in oncology: drug development cases leading by example. Clin Cancer Res. 2016;22(6):1318-24.

9. Venkatakrishnan K, Friberg LE, Ouellet D, et al. Optimizing oncology therapeutics through quantitative translational and clinical pharmacology: challenges and opportunities. Clin Pharmacol Ther. 2015;97(1):37-54.

10. Gupta N, Diderichsen P, Hanley M, et al. Population pharmacokinetic analysis of ixazomib, an oral proteasome inhibitor, including data from the phase III TOURMALINE-MM1 study to inform labelling. Clin Pharmacokinet. Epub 2017 Mar 13. doi:10.1007/ s40262-017-0526-4

11. Gupta N, Zhao Y, Hui AM, et al. Switching from body surface areabased to fixed dosing for the investigational proteasome inhibitor ixazomib: a population pharmacokinetic analysis. Br J Clin Pharmacol. 2015;79(5):789-800.

12. Gupta N, Goh YT, Min CK, et al. Pharmacokinetics and safety of ixazomib plus lenalidomide-dexamethasone in Asian patients with relapsed/refractory myeloma: A phase 1 study. J Hematol Oncol. 2015;8:103.

13. Kumar SK, Bensinger WI, Zimmerman TM, et al. Phase 1 study of weekly dosing with the investigational oral proteasome inhibitor ixazomib in relapsed/refractory multiple myeloma. Blood. 2014;124(7):1047-55.

14. Kumar SK, Berdeja JG, Niesvizky R, et al. Safety and tolerability of ixazomib, an oral proteasome inhibitor, in combination with lenalidomide and dexamethasone in patients with previously untreated multiple myeloma: An open-label phase 1/2 study. Lancet Oncol. 2014;15(13):1503-12.

15. Kumar SK, LaPlant B, Roy V, et al. Phase 2 trial of ixazomib in patients with relapsed multiple myeloma not refractory to bortezomib. Blood Cancer J. 2015;5:e338. 
16. Kumar SK, LaPlant B, Reeder CB, et al. Randomized phase 2 trial of two different doses of ixazomib in patients with relapsed multiple myeloma not refractory to bortezomib. Blood. 2015;126(23):3050.

17. Richardson PG, Hofmeister CC, Rosenbaum CA, et al. Twiceweekly oral MLN9708 (ixazomib citrate), an investigational proteasome inhibitor, in combination with lenalidomide (len) and dexamethasone (dex) in patients (pts) with newly diagnosed multiple myeloma (MM): final phase 1 results and phase 2 data. Blood. 2013;122(21):535

18. Richardson PG, Baz R, Wang M, et al. Phase 1 study of twiceweekly ixazomib, an oral proteasome inhibitor, in relapsed/ refractory multiple myeloma patients. Blood. 2014;124(7):1038-46.

19. Rajkumar SV, Dimopoulos MA, Palumbo A, et al. International myeloma working group updated criteria for the diagnosis of multiple myeloma. Lancet Oncol. 2014;15(12):e538-48.

20. Sanchorawala V, Palladini G, Kukreti V, et al. A phase $1 / 2$ study of the oral proteasome inhibitor ixazomib in relapsed or refractory $\mathrm{AL}$ amyloidosis. Blood. 2017;130(5):597-605.

21. Kumar S, Moreau P, Hari P, et al. Management of adverse events associated with ixazomib plus lenalidomide/dexamethasone in relapsed/refractory multiple myeloma. $\mathrm{Br} \mathrm{J}$ Haematol. 2017;178(4):571-82.

22. Benboubker L, Dimopoulos MA, Dispenzieri A, et al. Lenalidomide and dexamethasone in transplant-ineligible patients with myeloma. N Engl J Med. 2014;371(10):906-17.

23. Hideshima T, Richardson P, Chauhan D, et al. The proteasome inhibitor PS-341 inhibits growth, induces apoptosis, and overcomes drug resistance in human multiple myeloma cells. Cancer Res. 2001;61(7):3071-6.

24. Mitsiades N, Mitsiades CS, Poulaki V, et al. Molecular sequelae of proteasome inhibition in human multiple myeloma cells. Proc Natl Acad Sci U S A. 2002;99(22):14374-9.

25. Mitsiades N, Mitsiades CS, Poulaki V, et al. Apoptotic signaling induced by immunomodulatory thalidomide analogs in human multiple myeloma cells: therapeutic implications. Blood. 2002;99(12):4525-30.

26. Das DS, Ray A, Song Y, et al. Synergistic anti-myeloma activity of the proteasome inhibitor marizomib and the IMiD immunomodulatory drug pomalidomide. Br J Haematol. 2015;171(5):798-812.
27. Chauhan D, Singh AV, Ciccarelli B, et al. Combination of novel proteasome inhibitor NPI-0052 and lenalidomide trigger in vitro and in vivo synergistic cytotoxicity in multiple myeloma. Blood. 2010;115(4):834-45.

28. Bianchi G, Richardson PG, Anderson KC. Promising therapies in multiple myeloma. Blood. 2015;126(3):300-10.

29. Chauhan D, Tian Z, Zhou B, et al. In vitro and in vivo selective antitumor activity of a novel orally bioavailable proteasome inhibitor MLN9708 against multiple myeloma cells. Clin Cancer Res. 2011;17(16):5311-21.

30. Durie BG, Hoering A, Abidi MH, et al. Bortezomib with lenalidomide and dexamethasone versus lenalidomide and dexamethasone alone in patients with newly diagnosed myeloma without intent for immediate autologous stem-cell transplant (SWOG S0777): a randomised, open-label, phase 3 trial. Lancet. 2017;389(10068):519-27.

31. Stewart AK, Rajkumar SV, Dimopoulos MA, et al. Carfilzomib, lenalidomide, and dexamethasone for relapsed multiple myeloma. N Engl J Med. 2015;372(2):142-52.

32. Lonial S, Waller EK, Richardson PG, et al. Risk factors and kinetics of thrombocytopenia associated with bortezomib for relapsed, refractory multiple myeloma. Blood. 2005;106(12):3777-84.

33. Lonial S, Richardson PG, San MJ, et al. Characterisation of haematological profiles and low risk of thromboembolic events with bortezomib in patients with relapsed multiple myeloma. $\mathrm{Br} \mathrm{J}$ Haematol. 2008;143(2):222-9.

34. Gupta N, Hanley MJ, Venkatakrishnan K, et al. Pharmacokinetics of ixazomib, an oral proteasome inhibitor, in solid tumour patients with moderate or severe hepatic impairment. Br J Clin Pharmacol. 2016;82(3):728-38.

35. Gupta N, Hanley MJ, Harvey RD, et al. A pharmacokinetics and safety phase $1 / 1 \mathrm{~b}$ study of oral ixazomib in patients with multiple myeloma and severe renal impairment or end-stage renal disease requiring haemodialysis. Br J Haematol. 2016;174(5):748-59.

36. Gupta N, Labotka R, Liu G, et al. Exposure-safety-efficacy analysis of single-agent ixazomib, an oral proteasome inhibitor, in relapsed/ refractory multiple myeloma: dose selection for a phase 3 maintenance study. Investig New Drugs. 2016;34(3):338-46. 